Supporting information for

On-Surface Crystallization Behaviors of H-Bond Donor-Acceptor Complexes at

\title{
Liquid/Solid Interfaces
}

Linxiu Cheng, ${ }^{1,6, " ~ B i n ~ T u, ~}{ }^{2, ~ " X u n w e n ~ X i a o, ~}{ }^{3, ~ " B e a t r i z ~ F e r i n g a ́ n, ~}{ }^{4}$ Raquel Giménez,

Xiaokang Li, ${ }^{5}$ Qiaojun Fang, ${ }^{2}$ Teresa Sierra, ${ }^{4, *}$ Yibao Li, ${ }^{5, *}$ Qingdao Zeng,,${ }^{\text {, }}$ Chen Wang $^{2, *}$

${ }^{1}$ CAS Key Laboratory of Standardization and Measurement for Nanotechnology, CAS Center for Excellence in Nanoscience, National Center for Nanoscience and Technology (NCNST), Beijing 100190, P. R. China. E-mail: zengqd@ nanoctr.cn.

${ }^{2}$ CAS Key Laboratory for Biomedical Effects of Nanomaterials and Nanosafety, CAS Center for Excellence in Nanoscience, National Center for Nanoscience and Technology, Beijing 100190, P. R. China. E-mail: wangch@ nanoctr.cn.

${ }^{3}$ College of Chemical Engineering, Ningbo University of Technology Ningbo, P. R. China.

${ }^{4}$ Departamento de Química Orgá nica, Instituto de Ciencia de Materiales de Aragón (ICMA), Facultad de Ciencias, Universidad de Zaragoza-CSIC, 50009 Zaragoza, Spain. E-mail: tsierra@unizar.es.

${ }^{5}$ Key Laboratory of Organo-pharmaceutical Chemistry, Gannan Normal University, Ganzhou 341000, P. R. China. E-mail: liyb@gnnu.cn.

${ }^{6}$ University of Chinese Academy of Sciences, Beijing 100049, P. R. China

1. Method of STM image calibration

2. STM images of self-assembly structures 
1. Method of STM image calibration

In this work, all the STM images are calibrated by comparing self-assembled images with atomically resolved HOPG images, where HOPG is considered as standard sample for STM calibration. Here, taking the calibration of A-TPC4 an example, to discript the calibration detail. STM image in Figure S3a is recorded at ambient condition with the imaging constants : $I_{\mathrm{set}}=445.6 \mathrm{pA}, V_{\text {bias }}=607.3 \mathrm{mV}$ at the scale of $10.1 \times 10.1 \mathrm{~nm}^{2}$. When the voltage and current are adjusted to graphite conditions $\left(I_{\text {set }}\right.$ $=955.0 \mathrm{pA}, V_{\text {bias }}=45.0 \mathrm{mV}$ ) in situ, HOPG image is obtained (Figure S3b). The lattice constant of HOPG are known precisely with the value of $a=2.46 \AA$, $b=2.46$ $\AA, \alpha=120^{\circ}$. On basis of the lattice constant of HOPG, the STM image could be calibrated and the unit cell of the assembled structure could be determined.

2. STM images of self-assembly structures

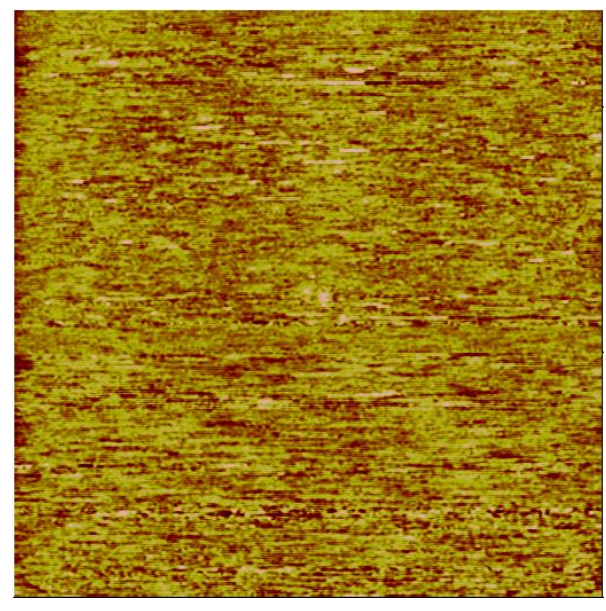

Figure S1. STM images of self-assembly structures for A-TPC6: at large scale (60.0 $\times 60.0) \mathrm{nm}^{2}, I_{\text {set }}=450.1 \mathrm{pA}, V_{\text {bias }}=560.4 \mathrm{mV}$. 


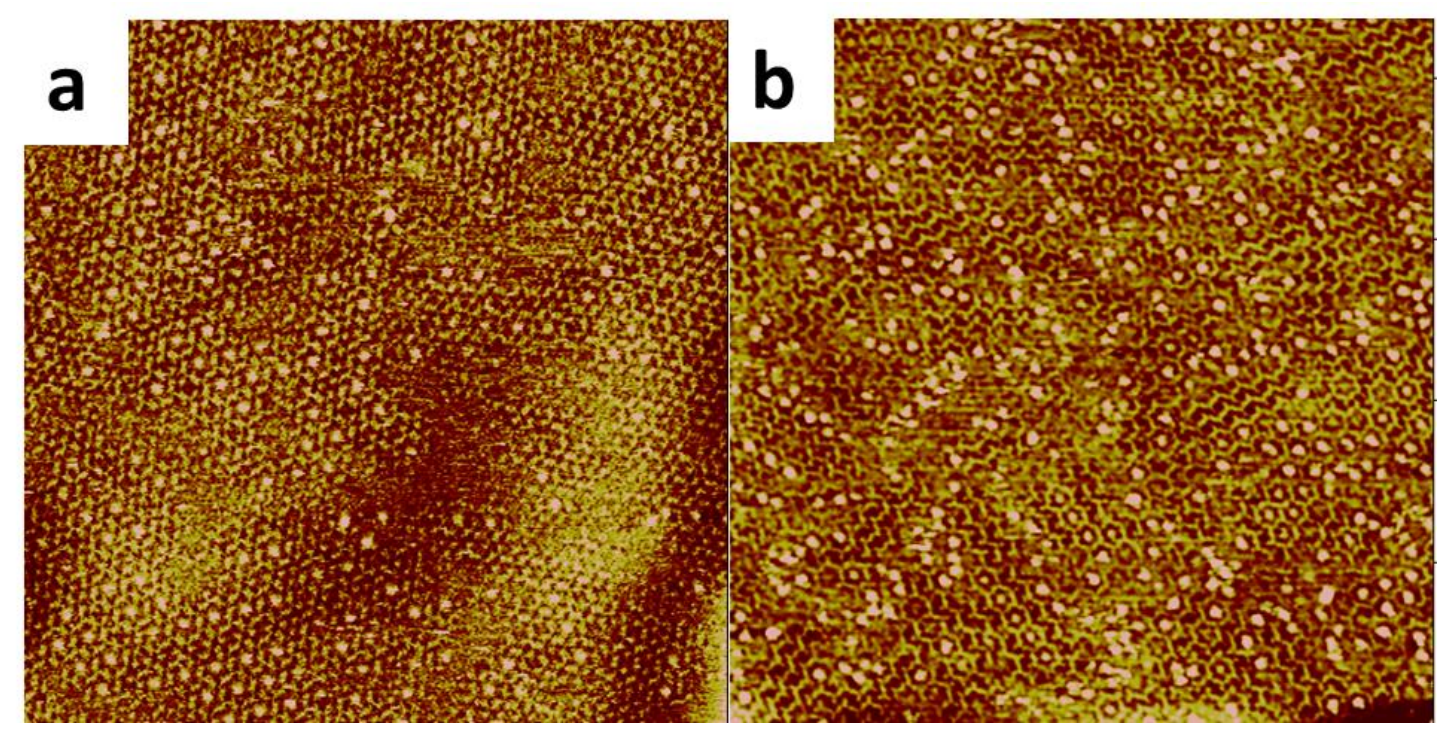

Figure S2. STM images of self-assembly structures for $\mathbf{A}-\mathbf{T P C} \mathbf{C}_{\mathbf{n}} / \mathbf{T} \mathbf{3} \mathbf{C}_{\mathbf{4}}$ systems: (a) A-TPC $4 /$ T3C $_{4}$ pattern at large scale $(87.1 \times 87.1) \mathrm{nm}^{2}, I_{\text {set }}=405.9 \mathrm{pA}, V_{\text {bias }}=501.4$ $\mathrm{mV}$. (b) A-TPC6/T3C4 pattern at large scale $(109.3 \times 109.3) \mathrm{nm}^{2}, I_{\text {set }}=454.7 \mathrm{pA}, V_{\text {bias }}$ $=556.9 \mathrm{mV}$.
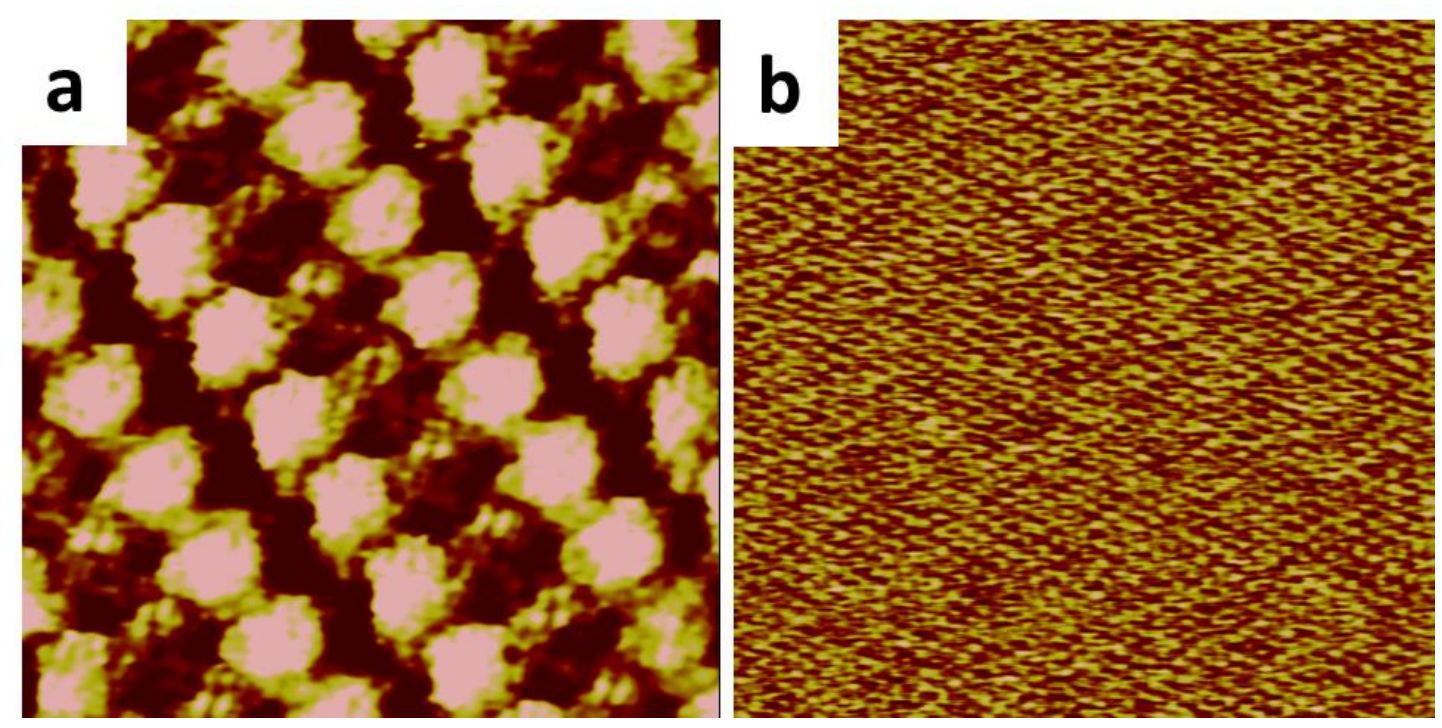

Figure S3. STM images of A-TPC 4 and HOPG: (a) of A-TPC 4 at the scale of $10.1 \times$ $10.1 \mathrm{~nm}^{2}, I_{\text {set }}=445.6 \mathrm{pA}, V_{\text {bias }}=607.3 \mathrm{mV}$. (b) HOPG image at the scale of $10.1 \times$ $10.1 \mathrm{~nm}^{2}$ (in situ), $I_{\text {set }}=955.0 \mathrm{pA}, V_{\text {bias }}=45.0 \mathrm{mV}$. 